Konzepte und Perspektiven 


\title{
Diesseits und jenseits der Grenze - das Konzept der Grenzregion
}

\section{Martin Klatt}

\begin{abstract}
Grenzregionen sind als oft wirtschaftlich periphere, aber im Geschichtsverständnis des $\mathrm{Na}$ tionalstaats symbolisch zentrale Regionen ein interessanter Forschungsgegenstand. Mit der europäischen Integration rückt die Vorstellung von der Grenzregion als Laboratorium dieser Integration in den Vordergrund. Heute gibt es an allen Grenzen Europas Formen der organisierten territorialen Zusammenarbeit in grenzüberschreitenden Regionen. Ihre Institutionalisierung und Tragweite sind jedoch sehr unterschiedlich. Dieser Beitrag wird Grenz- und grenzüberschreitende Regionen diskutieren, sowohl politisch-territorial als auch mit dem Fokus auf die Menschen in Grenzregionen und ihr soziales Verhalten.
\end{abstract}

\section{Schlagwörter}

Region, Grenzregion, grenzüberschreitende Zusammenarbeit, grenzüberschreitende Region

\section{Einleitung}

Der folgende Beitrag schildert, warum es sinnvoll für die Human- und Sozialwissenschaften ist, sich mit Grenzregionen zu befassen. Nach einer Begriffsklärung (Region, Grenzregion, grenzüberschreitende Region) werden verschieden Forschungsansätze zur Analyse von Grenzund grenzüberschreitenden Regionen und ihrer Einwohner*innen vorgestellt und verglichen. Kernfrage ist, inwieweit Grenzen das Verhalten und die Identität von Menschen beeinflussen und umgekehrt. In einem abschließenden Ausblick wird die Thematik in Bezug zur europäischen Integration und ihrem Ziel des Überwindens von Grenzen gesetzt.

Eine Grenze ist zunächst eine eindeutig markierte geographische Linie, die zwei souveräne Staaten trennt. Grenzen repräsentieren die Bedeutung von Territorialität als modernem Prinzip der Ordnung von politischem und sozialem Leben (vgl. Anderson/O'Dowd 1999). Darüber hinaus ist die Grenze der Rand, die Peripherie, eines oft als relativ homogen aufgefassten Gebietes bzw. Untersuchungsraums. Neben der kartographischen Festlegung der Grenze interessiert sich die human- und gesellschaftswissenschaftliche Grenzforschung vor allem für die Beziehung von Grenzen zu den Menschen und der Gesellschaft sowie die Frage, in welcher Form und mit welcher Funktion Grenzen produziert, überwunden und reproduziert werden (vgl. Anderson 1996; Donnan/Wilson 1999; Brambilla et al. 2015).

Die Grenzregion dient als Untersuchungsraum dieser Fragen (vgl. Banse 2004). Bei der räumlichen Abgrenzung einer Grenzregion greift man der Einfachheit halber meist auf existierende administrative Gliederungen der jeweiligen Staaten beiderseits der Grenze zurück. Seltener wird ein absolutes geographisches Entfernungskriterium (zum Beispiel bis zu 50 bis $100 \mathrm{~km}$ Luftlinie von der Grenze) benutzt oder eine funktionale Grenzregion gewählt, welche sich auf Handelsströme, Netzwerke und andere grenzbezogene soziale Aktivitäten bezieht (vgl. Blatter 2004). 


\section{Region, Grenzregion und grenzüberschreitende Region}

Der Begriff Region, eine Entlehnung aus dem Lateinischen regio (territoriales Gebiet) bzw. regere (leiten, dirigieren bzw. regieren), verbindet die territoriale Gliederung eines Raums mit der Organisation menschlicher bzw. gesellschaftlicher Herrschaft, Ordnung und Verwaltung. Alltagssprachlich wird der Begriff vor allem naturgeographisch definiert, zum Beispiel als Bodenseeregion, Harz, Flachland, Bergregion oder auch Nord- bzw. Süddeutschland; ohne eindeutige geographische Abgrenzung. Strukturell macht es Sinn, zwischen eher ländlich und eher städtisch geprägten Regionen zu unterscheiden, aber auch zwischen wirtschaftlich und politisch zentralen und peripheren Regionen (vgl. Fitjar 2010). In den Gesellschaftswissenschaften haben sich jedoch seit den 1990er-Jahren Bemühungen durchgesetzt, den Begriff Region zu operationalisieren und als räumlichen Gegenbegriff zum Staat bzw. Nationalstaat zu benutzen. Region kann dabei subnational, also als Untergliederung eines Staates, aber auch supranational als geographischer Großraum verstanden werden (z.B. die EU als Region oder Skandinavien bzw. der ,Norden`). Für das Thema Grenzregion sind Letztere aber nicht relevant.

Regionen sind seit 1993 mit dem Ausschuss der Regionen in den Gesetzgebungsprozess der EU eingebunden. Auch sind seither in vielen EU-Mitgliedsstaaten Regionen institutionalisiert und in ihren Kompetenzen gestärkt worden. Die Forschung hat diese Entwicklungen unter dem Begriff New Regionalism zusammengefasst, der Regionen als erstarkende politische Akteure sowohl in der Industrie- und Wirtschaftspolitik (vgl. Ohmae 1995; Keating 2004) als auch in der Außenpolitik (vgl. Aldecoa/Keating 1999; Klatt/Wassenberg 2018) entdeckt hat.

Am weitesten entwickelt ist das Konzept Region bei Anssi Paasi (2002) und seiner Unterscheidung, von vier verschiedenen Merkmalsausprägungen (shapes) einer Region zu sprechen: ihrer territorialen, symbolischen, institutionellen und identitären Gestalt. Paasi versteht Regionen als zunächst historisch bedingte politische Strukturen, deren soziale Konstruktion auf der Institutionalisierung ihrer territorialen, symbolischen und institutionellen Gestalt beruht. Die identitäre Gestalt der Region ist eng mit dem Prozess der Institutionalisierung verbunden, der von Paasi als entscheidend für die Entwicklung einer regionalen Identität und Identifikation der Bewohner"innen angesehen wird. Analog zum Konsens in der sozialkonstruktivistischen Nationalismusforschung (vgl. Hobsbawm/Ranger 1983; Hroch 2005) wird den regionalen Eliten eine zentrale Rolle in diesem Prozess zugeschrieben (vgl. Paasi 2009b). Ebenso bedarf die institutionalisierte Region eines abgegrenzten Territoriums und einer erkennbaren Symbolik (z.B. Flagge, historische Mythen, volkskulturelle Symbole wie Trachten oder regionale Speisen) als Voraussetzung für die Bildung von Identität. Aus politikwissenschaftlicher Perspektive ist jedoch, trotz größerer regionaler Autonomie und interregionaler Vernetzung, auch heute der Staat der entscheidende Organisator und Identitätsbilder des territorialen Raumes (vgl. Paasi 2009a).

Grenzregionen verbinden regionale Konzepte mit dem Staatskonzept, da sie mehr als eine staatliche Untergliederung darstellen. Staatsgrenzen sind das Resultat historischer Prozesse der Herrschaftssicherung und territorialen Ordnung des modernen Staates (vgl. Donnan/Wilson 1999). Diese Prozesse betreffen sowohl Lage als auch Funktion der Grenze. Damit sind Grenzen eine politische Konstruktion mit realen Konsequenzen. Sie basieren auf dem Souveränitätsprinzip, der Nichteinmischung in die inneren Angelegenheiten eines Staates. Souveränität verlangt definierte und markierte territoriale Grenzen sowie das Recht und die Fähigkeit des 
Staates, diese und die grenzüberschreitenden sozialen Prozesse zu kontrollieren (vgl. Ratzel 1901/2017). Hierzu gehört auch der staatliche Anspruch der Homogenisierung von Territorium, Identität und politischer Gemeinschaft (vgl. Kolossov/Scott 2013). Dies hat entscheidenden Einfluss auf Grenzregionen. Mit der Entwicklung der wirtschaftlichen, normativen und sozialen Funktion von Grenzen im modernen Staat wurden vor allem im 19. und der ersten Hälfte des 20. Jahrhunderts Wirtschafts- und Kulturräume getrennt. Die Bevölkerung hat sich in der Mehrzahl diesen neuen Gegebenheiten angepasst, etwa durch sprachliche Assimilation, aber auch durch Veränderung der Alltagsgewohnheiten. Die Europäische Integration versucht seit den 1950er-Jahren, diese Entwicklung rückgängig zu machen und Grenzen zu überwinden. Damit sind Grenzen auch Anwendung und Gewohnheit (social practices), situiert und konstituiert in politischer Verhandlung und ihrem Gebrauch im täglichen Leben (vgl. Andersen/Sandberg 2012).

Dies bedeutet, dass die Grenzregion ein Ort der Konfrontation, aber auch der Begegnung mit dem Anderen ist, wo das Eigene in Frage gestellt wird. Neben der symbolischen Bedeutung gibt es eine reale wirtschaftliche Konsequenz der Grenze. Grenzen stellen eine Barriere des freien Handels dar, die Kosten verursacht, etwa für Zölle, Abgaben, Wartezeiten bei Kontrollen, aber auch Markteindringungskosten wie zum Beispiel divergierende technische Standards, Übersetzungskosten für Betriebsanleitungen, Aufbau einer Verkaufsorganisation im Nachbarland. Grenzregionen gelten deshalb als wirtschaftlich benachteiligt und weisen oft verhältnismäßig schlechte sozioökonomische Kennziffern auf. Allerdings kann sich in Grenzregionen auch eine spezifische Grenzökonomie ansiedeln. So können sich bestimmte Unternehmen mit Vorteil in einer Grenzregion ansiedeln, um beispielsweise von unterschiedlichen staatlichen Abgaben, einem unterschiedlichen Lohnniveau oder anderer Faktoren zu profitieren, wie auch die Bewohner einer Grenzregion von besseren Verdienstmöglichkeiten im Nachbarland oder einem günstigeren Preisniveau für Nahrungs- und Genussmittel bzw. Immobilien profitieren können. Man verwendet deswegen auch den Begriff Grenze als Ressource (vgl. Sohn 2014).

Mit der Europäischen Integration stellte sich schon in den Anfängen mit Ende der 1950er-Jahre die Frage der Bildung grenzüberschreitender Regionen als Instrument der lokalen Zusammenarbeit in einem Europa von unten (vgl. Eigmüller in diesem Band). Über die Errichtung von Euroregionen wurde seit damals die grenzüberschreitende Region operationalisiert. Euroregionen sollen ein Zusammengehörigkeitsgefühl der Bürger*innen entlang der Grenze erzeugen bzw. verstärken und den institutionellen Rahmen für weitgehende kommunalpolitische, kulturelle und wirtschaftliche Zusammenarbeit bereitstellen. Sie entstanden bis in die 1970erJahre an allen gemeinsamen Grenzen der EG-Gründungsmitglieder. Die zweite Phase der Gründung von Euroregionen war das erste Jahrzehnt nach dem Ende des Kalten Krieges, als insbesondere an den Grenzen der ehemaligen Mitgliedsstaaten des Warschauer Vertrags neue Euroregionen entstanden. Auch in Skandinavien wurden nun die oft schon seit den 1960erund 1970er-Jahren bestehenden losen grenzüberschreitenden Arbeitsgemeinschaften institutionalisiert, wenn auch ohne den Begriff Euroregion zu verwenden. Im ersten Jahrzehnt des 21. Jahrhunderts kamen schließlich die letzten Euroregionsgründungen hinzu, nicht zuletzt bedingt durch Interreg, das Förderprogram der EU für Grenzregionen (vgl. Durà et al. 2018). Heute befinden sich an allen Grenzen Europas, sowohl den Binnengrenzen der EU als auch ihren Außengrenzen und sogar an Grenzen zwischen Drittstaaten, Euroregionen. Zudem erschuf die EU 2006 mit der Möglichkeit des Rechtsinstruments Europäischer Verbund für Territoriale 
Zusammenarbeit (EVTZ, englisch: European Grouping of Territorial Cooperation, EGTC) einen formalen juristischen Rahmen für grenzüberschreitende Regionen.

\section{Forschungsansätze zur Analyse von Grenz- und grenzüberschreitenden Regionen}

Das folgende Kapitel stellt verschiedene Ansätze der Forschung vor, mit denen Grenz- und grenzüberschreitende Regionen verglichen und analysiert werden. Solche Analysen können historischer, politikwissenschaftlicher, geographischer, linguistischer, kultureller und regionalökonomischer Art sein oder einen interdisziplinären Ansatz verfolgen. Die Grenzregion steht dabei im Zusammenhang zur Grenze als Markeur staatlicher Souveränität im staatszentrierten Verständnis der westfälischen Ordnung, spiegelt aber gleichzeitig die relationale Komponente der Personen-, Kapital- und Warenströme der globalisierten Netzwerke wider, welche sich nicht unbedingt nach fixierten Grenzen richten (vgl. Kolossov/Scott 2013). Damit stellt sich die Frage, ob man Grenzregionen nur grenzüberschreitend sinnvoll untersuchen kann, da sie immer im Verhältnis zur Grenzregion auf der anderen Seite der Grenze stehen. Grundlegend relevant für eine Typologisierung von Grenzregionen ist deshalb immer noch Oscar Martinez' (1994) Unterscheidung von alienated, coexistent, interdependent und integrated borderlands. Der Charakter der Grenzregion ist demnach abhängig von den bilateralen Beziehungen der Staaten, um deren Grenze es sich handelt, und dem sich daraus folgenden Grad der Offenheit der Grenze für den Personen- und Warenverkehr. Dieses Modell analysiert spezifische grenzregionale Situationen und ist im Ansatz normativ, da Martinez den Zustand des integrated borderland mit andauernd stabilen politischen Verhältnissen an der Grenze, funktioneller Fusion der Volkswirtschaften beiderseits der Grenze, freiem Personen- und Warenverkehr und Einwohner"innen der Grenzregion, die sich als Mitglieder eines gemeinsamen sozialen Systems empfinden (ebd., S. 7), für erstrebenswert hält.

Peter Schmitt-Egner und Emmanuel Brunet-Jailly haben diesen teleologischen Ansatz weiterentwickelt. Schmitt-Egner (1998) hat ein dreizehnstufiges Modell entwickelt, an dessen Schlusspunkt die grenzüberschreitende Region steht, als ein von ihren politischen und wirtschaftlichen Akteuren und schließlich auch ihren Bewohnern empfundener einheitlicher, grenzüberschreitender Handlungsraum. Brunet-Jailly (2005) untersucht in seinem Modell zur Bildung grenzüberschreitender Regionen in vormals relativ unabhängig voneinander operierenden Regionen den Zusammenhang zwischen funktionalen Strömen und territorialen Konzepten. Nach Brunet-Jailly sind vier gegenseitig abhängige und sich befruchtende analytische Linsen („lenses“, ebd., S. 633) für die Evolution einer grenzüberschreitenden Region entscheidend: eine lokale, grenzüberschreitende Kultur, eine lokale, grenzüberschreitende politische clout (wahrscheinlich am besten mit „Einflusssphäre“ übersetzt), grenzüberschreitende multi-level governance sowie grenzüberschreitende Marktkräfte und Handelsströme (vgl. dazu Hungerland/Teupe in diesem Band). Brunet-Jailly setzt diese vier Linsen unabhängig miteinander in Beziehung, d.h. keine wird als erste Grundvoraussetzung und Startpunkt der grenzüberschreitenden Regionsbildung definiert. Damit ermöglicht das Modell sowohl einen funktionalen Ansatz, in dem die grenzüberschreitenden Marktkräfte, Handelsströme oder aber auch Umweltprobleme zur Institutionalisierung einer grenzüberschreitenden politischen clout führen, oder aber umgekehrt einen territorialen Ansatz, in dem die politische Institutionenbildung grenzüberschreitende Handelsströme ermöglicht oder verstärkt. 
Die Schwäche dieser teleologischen Konzepte ist die bis heute fehlende empirische Nachweisbarkeit des Endzustands der integrierten grenzüberschreitenden Region. Fallstudien bestätigen die nach wie vor wirtschaftlich und sozial abgrenzende Funktion der Grenze auch in Grenzregionen innerhalb der EU. Es gibt aber beträchtliche Unterschiede im Integrationsgrad grenzüberschreitender Regionen. Die bisher umfassendste sozioökonomische Untersuchung wurde 2004 von 387 NUTS-3-Regionen ${ }^{1}$ an den damaligen Grenzen der EU (exklusive Malta und Zypern) sowie den Grenzen der damaligen Beitrittskandidaten Rumänien und Bulgarien durchgeführt. Sie demonstriert vor allem die Unterschiedlichkeit sozioökonomischer Verhältnisse in europäischen Grenzregionen. Das Forschungsteam um Lefteris Topaloglu (vgl. Topaloglou et al. 2005) hat mit Hilfe einer Fuzzy-Cluster-Analyse ${ }^{2}$ fünf Cluster von Grenzregionstypen kategorisiert (ebd., S. 84):

„A: Highly integrated border regions with advanced economic performance, many cultural similarities and small size (Border regions in the EU15 core, Scandinavia, Ireland, UK)

B: Border regions that enjoy agglomeration economies but need significant structural adjustments in order to deal with the increased competition (Border regions in the Baltics, Slovakia, Czech Republic, Poland)

C: Highly integrated border regions that present significant economic performance, though much cultural dissimilarity (Border regions in France, Germany, Spain, Portugal, Italy and Austria)

D: Border regions with high development potential due to their favorable geographic position, but with low economic performance (Border regions on the western side of EU new member states)

E: Border regions with low market potentials and no prevailing positive characteristics (Border regions in the EU external borders prior to 2004-enlargement)“

Diese erste systematisch erarbeitete Typologisierung von Grenzregionen stellte fest, dass die Häufigkeit von Interaktion über die Grenze nicht unbedingt die Konvergenz der Regionen beiderseits der Grenze zur Folge hat. Ein weiteres Ergebnis war aber auch, dass die nationalen Teile grenzüberschreitender Regionen der westlichen Grenzregionen ausnahmslos im gleichen Cluster dominieren, während ein Gegensatz der Typologien in den nationalen Teilen grenzüberschreitender Regionen der ,neuen“ EU-Mitglieder deutlich sichtbar wurde. Der europäische Integrationsprozess in den Kernländern der EU hat also durchaus zu Konvergenz in ihren Grenzregionen geführt. Andere Fallstudien bestätigen, dass Grenzen weiterhin einen signifikanten ökonomischen Effekt haben: Grenzregionen bleiben unter ihrem ökonomischen Potenzial, so wie auch regionale Spill-over-Effekte im Wissens- und Technologietransfer weiterhin durch Grenzen behindert werden (vgl. Naveed/Ahmad 2016; Makkonen et al. 2018). Die Häufigkeit grenzüberschreitender Interaktionen korreliert in vielen Grenzregionen mit sozioökonomischen Unterschieden, aber beseitigt sie nicht (vgl. Sohn/Lara-Valencia 2013).

Untersuchungsgegenstand der vergleichenden Forschung zu grenzüberschreitenden Regionen sind vor allem die territorial abgegrenzten Euroregionen (vgl. Scott 2000; Perkmann 2002; Heddebaut 2004; Lepik 2009; Lewandowski/Greta 2010; Medeiros 2011; Durà et al. 2018).

1 NUTS (französisch: nomenclature des unités territoriales statistiques) ist eine statistische geographisch-administrative Einheit der Europäischen Union. In Deutschland entspricht NUTS 3 den Landkreisen.

2 Fuzzy Clustering ist eine statistische Methode, in der jeder Datenpunkt nicht nur einem Cluster (also einer Häufung) zugeordnet wird, sondern mehreren zugeordnet werden kann. 
Markus Perkmann (2003, S. 157) definiert Euroregionen als territoriale grenzüberschreitende Regionen: „a bounded territorial unit composed of the territories of authorities participating in a CBC initiative." Die Glieder können Provinzen, Bundesländer, Kantone, Kreise, Kommunen sein. Euroregionen beruhen auf einem Abkommen der Partner, jedoch nicht auf völkerrechtlich bindenden Verträgen. Eine grenzüberschreitende Region ist damit eine sozialterritoriale Einheit mit einem gewissen Grad an strategischer Kapazität und Organisation. Territoriale Abgrenzungen grenzüberschreitender Regionen sind aber oft willkürlich und politisch konstruiert. So ist zum Beispiel die von der EU definierte Größe des Interreg-Fördergebiets ein Anreiz, sich einer grenzüberschreitenden Euroregion anzuschließen. Historisch lässt sich hier eine Fluktuation feststellen, da die Mitgliedschaft in einer Euroregion nach politischer Interessenlage und Fördermöglichkeiten gewählt wird (vgl. z.B. Medve-Balint/Svensson 2012).

Vorübergehend wurden Euroregionen aber durchaus als ein Zeichen einer Reterritorialisierung im post-westfälischen subnationalen Europa eingeschätzt (z.B. Popescu 2008). Gabriel Popescu (ebd.) demonstriert, dass geopolitische Ambitionen von Staaten und Regionen in Osteuropa durchaus eine wichtige Rolle bei der Errichtung von Euroregionen gespielt haben. Diese haben für politischen Sprengstoff in bilateralen Beziehungen gesorgt, da es in Staaten wie Ungarn und Rumänien politische Kräfte gibt, die mit dem Gedanken von Grenzrevisionen spielen. Dies wurde insbesondere bei der Errichtung der Euroregion Karpatien (Ungarn, Polen, Ukraine und Rumänien) deutlich (ebd.), bei der sich die rumänische Zentralregierung übergangen fühlte. Die regionalen rumänischen Vertreter, die an den Feierlichkeiten zur Errichtung der Euroregion teilgenommen hatten, wurden ihrer Ämter enthoben. Auch an der deutsch-dänischen Grenze gab es Bedenken einer Einmischung Deutschlands in innere dänische Angelegenheiten im Falle der Einrichtung einer Euroregion (vgl. Klatt 2006; Yndigegn 2012).

Eine wiederkehrende Frage im Zusammenhang mit der politischen Etablierung grenzüberscheitender Regionen ist die nach ihrer Wirkmächtigkeit. Bisher existieren nur wenige breit angelegte Studien zur Analyse der Wirkmächtigkeit grenzüberschreitender Regionen in Europa. Als Variable wird dabei häufig der Aktivitätsgrad grenzüberschreitender Regionen gewählt: Perkmann hat um die Jahrtausendwende die Intensität der Zusammenarbeit in 74 grenzüberschreitenden Regionen (güR) anhand eines Katalogs der Association of European Border Regions (AEBR) analysiert und diese dann in vier Kategorien eingeteilt (vgl. Perkmann 2003): integrierte mikro-güR, entstehende mikro-güR, skandinavische Gruppierungen und großräumige Arbeitsgemeinschaften. Mikro-güR entsprechen den klassischen, institutionalisierten Euroregionen, großräumige Arbeitsgemeinschaften sind eine Kategorie eher lose institutionalisierter grenzüberschreitender Zusammenarbeit. Für die nur schwach institutionalisierten skandinavischen grenzüberschreitenden Regionen wählt Perkmann die Sonderkategorie skandinavische Gruppierungen, um auszudrücken, dass sie trotz eines geringen Institutionalisierungsgrades intensiv zusammenarbeiten. Es konnte somit kein notwendiger Zusammenhang zwischen dem Aktivitätsgrad und dem Grad der Institutionalisierung einer güR nachgewiesen werden. Gleiches bestätigt Eduardo Medeiros' Vergleich schwedisch-norwegisch und spanisch-portugiesischer grenzüberschreitender Regionen (2011). Hier sind es eher die Konstanz in der Kooperation sowie funktionale Elemente wie räumliche Nähe, Infrastruktur und Verdichtung, welche die Intensität der Zusammenarbeit verstärken.

Wirkmächtigkeit liegt auch dem jüngsten Versuch einer Typologisierung von grenzüberschreitenden Regionen zu Grunde. In einem mehrjährigen Projekt der Autonomen Universität Barcelona wurde auf der Basis einer Aktivitätsüberprüfung von Euroregionen und der Auswertung 
der während der Interreg IV-Förderperiode (2007-2013) unterstützten Projekte eine Bewertung von Strukturen territorialer Zusammenarbeit erstellt und in einem Katalog von good practices in den Kriterien Exzellenz und Innovation veröffentlicht (vgl. Durà et al. 2018). Entscheidend für die Aufnahme in den Katalog waren dokumentierte Aktivitäten sowie die ausdrückliche Erklärung in der Charta bzw. Satzung der Euroregion, direkt oder indirekt an der Entwicklung und Durchführung grenzüberschreitender Projekte beteiligt zu sein.

Die oben angeführten Untersuchungen dokumentieren, dass grenzüberschreitende Integration nicht unbedingt mit historischen oder heutigen politisch inspirierten Raumkonzepten vor allem in der EU korreliert. Auch vor dem Hintergrund nordamerikanischer Erfahrungen rückten Grenzregionsforscher verstärkt die Waren- und Personenströme über Grenzen in den Vordergrund ihrer Empirie (siehe auch Schindler in diesem Band): der Fokus verschob sich von spaces of place, also territorial definierten Regionen, hin zu spaces of flow (vgl. Blatter 2004). Warenund Handelsströme waren auch im schon erwähnten Modell zur Bildung grenzüberschreitender Regionen von Brunet-Jailly (2005) eine entscheidende Variable. Die Region wird damit eher relational, also als Beziehungsgeflecht von Akteuren, als territorial verstanden (vgl. Varró/ Lagendijk 2012). Funktionale grenzüberschreitende Regionen können entlang von Infrastrukturachsen bzw. Transportkorridoren definiert werden, wie die Oberrhein-Region (DE-FR-CH; vgl. Zumbusch/Scherer 2015) oder der Jütlandskorridor (DE-DK; Danish-German 2015), oder als Einzugsräume grenzüberschreitender Metropolregionen (z.B. die Grande Région um Luxemburg, vgl. Wille 2015).

Die Idee der Euroregion als einer Reterritorialisierung und Entgrenzung Europas mit neuen oder wiederentdeckten grenzüberschreitenden Regions- und Identitätskonzepten bleibt damit eine politische Ambition (vgl. Scott 2013) oder dreamscape (vgl. Löfgren 2008), während sich grenzregionale Integration vor allem auf wenige funktionelle Aspekte beschränkt. Selbst diese Integration gleicht eher einer cooperation by bottleneck, in der gewisse ,Flaschenhälse' auf dem Arbeitsmarkt oder auch im Rettungswesen und anderen öffentlichen Diensten oft nur vorübergehend grenzüberschreitend gelöst werden, als dass dauerhaft gemeinsame funktionale Infrastruktur geschaffen wird (vgl. z.B. ESPON 2018). Es sind vor allem private Akteur*innen, die durch ihr Verhalten grenzüberschreitende Regionen als Aktionsräume schaffen; sei es durch Grenzhandel, durch Arbeits- und Wohnmobilität über die Grenze oder als Wirtschaftsunternehmen. Grenzüberschreitende Aktivitäten, Waren- und Handelsströme entwickeln sich anhand konkreter Anreize, während ein direkter Zusammenhang zum politischen Organisationsgrad und dem Grad der Institutionalisierung einer grenzüberschreitenden Region nicht notwendig festgestellt werden kann.

\section{Border Surfers und Regionaut*innen: Alltag der Grenzregionsbewohner*innen?}

Der vorangegangene Fokus auf die Struktur Grenz- und grenzüberschreitender Regionen soll nun um den Fokus auf die Bewohner"innen von Grenzregionen ergänzt werden. Wie nutzen diese Menschen die Grenzregion bzw. wie werden sie von der Grenze geprägt? Im Folgenden soll erläutert werden, wie der Blick auf die Menschen in der Grenzregion zum Verständnis dieser speziellen Regionen und ihrer Entwicklung beitragen kann.

Grenzen sind nicht nur eine Linie im Sand (vgl. Parker/Vaughan-Williams 2009), sondern ein Prozess: Sie sind von Menschen gemacht und beeinflussen das Handeln der Menschen, 
wie auch das Handeln der Menschen wiederum die Grenze beeinflusst (vgl. Wille in diesem Band). Der Begriff borderwork (vgl. Rumford 2008) beschreibt, wie die Grenze durch die Praxis bestimmt wird, wie sie Normen setzt und das Verhalten von Menschen prägt, aber auch wie Menschen durch ihre Praxis die Grenze definieren. Wie schaffen Menschen sich ihre borderscape (vgl. Brambilla 2015) bzw. wie werden sie durch diese beeinflusst? Am Beispiel afrikanischer und asiatischer Grenzen, wo die Anpassungsprozesse der Menschen an Grenzziehungen auch heute gut empirisch beobachtbar sind, haben Michiel Baud und Willem van Schendel (1997) demonstriert, wie Bewohner*innen von Grenzregionen die Grenze herausfordern. Sie ignorieren die Grenze, wenn es sich für sie lohnt, und nutzen die Vorteile der Grenze auf eine Weise, die von den ,Grenzziehern' (also den Staaten) so nicht vorgesehen war (ebd.). Gleichzeitig gibt es Prozesse der sozialen Adaption an die neuen Realitäten: Durch Einbindung lokaler Eliten können die Zentren der Staaten eine Änderung sozialer Praktiken wie wirtschaftlicher Handlungsmuster, aber zum Beispiel auch von Heiratsmustern erreichen, so dass die Grenze innerhalb weniger Generationen zunehmend von einer politischen auch zu einer sozialen Grenze wird (ebd.).

Oscar Martinez hat Bewohner*innen von Grenzregionen durch ihre Einteilung in national borderlanders und transnational borderlanders mit diversen Schattierungen in Beziehung zur Grenze gesetzt (1994). Während national borderlanders die Grenze selten überqueren und indifferent zu den Bewohner*innen und der Kultur des Nachbarlandes sind, haben transnational borderlanders enge Verbindungen zum Nachbarland und überqueren die Grenze häufig. Sie sehen die grenzüberschreitende Region als zusammenhängenden Lebens- und Handlungsraum. In der europäischen Grenzforschung wurde Martinez' Kategorisierung der Grenzregionsbewohner*innen durch die Begriffe Regionaut*innen (regionauts; vgl. O'Dell 2003) und Grenzsurfer*innen (border surfers; vgl. Terlouw 2012) ergänzt. Regionaut*innen, begrifflich abgeleitet von Astronaut*innen, sind Menschen, die Fähigkeiten entwickeln, um den Raum beidseits der Grenze aktiv nutzen zu können (vgl. Löfgren 2008). Der Begriff Grenzsurfer*innen soll deutlich machen, dass Grenzregionsbewohner"innen ihr Verhalten nicht unbedingt nach den Intentionen der EU-Förderprogramme oder den Visionen politischer Akteure einer grenzüberschreitenden Euroregion ausrichten. Oft sind es persönliche Bedürfnisse und Vorteile, die sich durch die Unterschiedlichkeit beiderseits der Grenze begründen (vgl. Klatt/Herrmann 2011; Terlouw 2012), die am Anfang einer Mobilität über die Grenze stehen: Arbeitslosigkeit und die Möglichkeit eines Jobs auf der anderen Seite, ein höheres Lohnniveau oder auch günstige Immobilienpreise.

Eine im Jahr 2015 in allen Interreg-Programmgebieten der EU-Mitgliedsstaaten durchgeführte Eurobarometer-Umfrage hat allerdings gezeigt, dass nur eine Minderheit der Einwohner*innen von Grenzregionen regelmäßig die Grenze überqueren (vgl. European 2015). Um Ursachen für die mangelnde Mobilität in europäischen Grenzregionen zu finden und zu erklären, haben Bas Spierings und Martin van der Velde (2008; 2013a) das Unfamiliarity-Konzept entwickelt. Dieses Konzept sieht Mobilität über die Grenze durch rationale Push- und Pull-Faktoren begründet, wie etwa Preisunterschiede, aber auch durch emotionale Faktoren wie ein exotisches Einkaufserlebnis. Gleichzeitig gibt es Keep-and-Repel-Faktoren, die Menschen von grenzüberschreitenden Aktivitäten zurückhalten. Der Grad der Familiarität beeinflusst das Wissen über grenzbedingte Unterschiede, aber auch das Unbehagen, sich zum Beispiel am Arbeitsplatz mit einer fremden Kultur auseinandersetzen zu müssen. Die Bandbreite der Nichtfamiliarität erklärt nun, wie rationale und emotionale Unterschiede, tatsächlich oder empfunden, die Ent- 
scheidungen beeinflussen, im Alltag für gewisse Aktivitäten die Grenze zu überschreiten oder auch nicht (vgl. Spierings/van der Velde 2008; 2013; Klatt 2014; Boese/Schnuer 2017). Diese Bandbreite ist bei einmaligen Mobilitätsaktivitäten wie einem Einkauf größer als bei dauerhafter Mobilität, wie etwa bei der Verlegung des Wohnsitzes oder Arbeitsplatzes ins Nachbarland. Soziale Netzwerke scheinen auf die Vergrößerung der Bandbreite der Nichtfamiliarität eine wichtige Rolle zu spielen: Sie können aus Einzelfällen von Regionaut*innen zur Entstehung von Gruppen von transnational borderlanders führen (vgl. Klatt 2014). Von Euroregionen verbreitete Images als grenzüberschreitende oder gar grenzenlose Region werden bei einer Analyse der sozialen Aktivitäten und Mobilitäten dann oft konterkariert, wenn diese offensichtlich einseitig durch klare Kostenvorteile erzeugt worden sind. Hierzu gibt es Beispiele insbesondere in den Regionen um Luxemburg, Basel und Genf, aber auch Kopenhagen, wo Metropolen als Magnet für Pendlerströme bzw. Umsiedlung ins Nachbarland wegen wesentlich niedrigerer Immobilienpreise fungieren (vgl. Terlouw 2008; Nerb et al. 2009).

Eine bisher in der Grenzregionsforschung kaum untersuchte Gruppe sind die in einigen Grenzregionen Europas existierenden nationalen Minderheiten. Insbesondere die Grenzziehungen nach dem Ersten Weltkrieg haben national diverse oder auch indifferente (vgl. Zahra 2010) Regionen geteilt. Als Teil des Friedensprozesses wurden nationale Minderheiten institutionalisiert, damit die Menschen, die sich national anders als die Titularnation des Residenzstaats identifizieren, als kollektiver, potenziell internationaler Akteur aufgefasst werden können und somit Relevanz für die politischen Prozesse in einer Grenzregion erhalten (vgl. Klatt 2017). Obwohl es in der Zwischenkriegszeit starke Anstrengungen der jeweiligen Staaten zu nationaler Homogenisierung gab und während und nach dem Zweiten Weltkrieg größere Bevölkerungsverschiebungen stattfanden, sind ethnisch nicht homogene Grenzregionen insbesondere für Zentral- und Osteuropa auch heute charakteristisch. Nationale Minderheiten können bei der Entwicklung der grenzüberschreitenden Zusammenarbeit eine wichtige Rolle als Brückenbauer*innen über die Grenze spielen (vgl. Malloy 2010). Gleichzeitig kann die Existenz institutionalisierter nationaler Minderheiten die Zusammenarbeit verkomplizieren, wenn Staaten Wünsche nach Grenzveränderungen oder Sezession fürchten. Dies ist zum Beispiel für Südtirol (vgl. Palermo 2005) und Sønderjylland-Schleswig (vgl. Klatt 2013; 2017) belegt. Als Individuen haben Mitglieder einer nationalen Minderheit durch ihre (vermeintliche) Zweisprachigkeit und Bikulturalität die besten Voraussetzungen, sich die Möglichkeiten einer Grenzregion wie den grenzüberschreitenden Arbeits- und Wohnraummarkt und Freizeitaktivitäten als Grenzsurfer*innen bzw. transnational borderlanders zu Nutze zu machen. Inwieweit dies passiert, ist bisher noch nicht genügend untersucht worden. Die existierenden Studien sehen aber noch erhebliches Potenzial, Minderheiten in die Erarbeitung grenzüberschreitender Regionalentwicklungsstrategien und konkreter grenzüberschreitender Projekte einzubeziehen (vgl. Klatt 2005; 2013; Klatt/Kühl 2008; Malloy 2010; Böhm/Drápela 2017).

\section{Ausblick}

Grenzregionen werden häufig als Laboratorien der europäischen Integration bezeichnet, da sich dort auch empirisch bestimmen lässt, in welchen Bereichen die Integrationsprozesse fortschreiten und wo sie behindert werden. Der sich entwickelnde Integrationsprozess, insbesondere die Verwirklichung des europäischen Binnenmarkts 1993 und die Abschaffung der permanenten Personenkontrollen mit dem Schengener Abkommen, haben Grenzregionen verändert und neue Möglichkeiten grenzüberschreitender Interaktion geschaffen. Das Interreg-Programm 
der EU hat diese Möglichkeiten seit 1991 gefördert und macht aber immer noch nur einen kleinen Teil der EU-Kohäsionspolitik aus. Heute existieren an allen europäischen Grenzen Strukturen der territorialen Zusammenarbeit, aber der Grad ihrer Intensität ist sehr unterschiedlich. Allen ist gemeinsam, dass sie institutionell schwach geblieben sind und eher als Rahmen von Netzwerken von Akteur*innen grenzüberschreitender Zusammenarbeit fungieren denn als eine neue Form territorialer und institutioneller Regionsbildung.

Die Entwicklung hin zu sich integrierenden grenzüberschreitenden Räumen wird derzeit von globalen Herausforderungen wie insbesondere dem Klimawandel und Migration, aber auch neuen Gefährdungen wie dem islamistischen Terrorismus, der COVID-19 Pandemie oder neuen Formen grenzüberschreitender Kriminalität und Steuerhinterziehung überschattet. Auch das Narrativ der EU als Erfolgsgeschichte wird hinterfragt: Der Brexit, aber auch das vorhandene wirtschaftliche Ungleichgewicht innerhalb der Staatengemeinschaft sowie das Erstarken rechtsund linkspopulistischer Bewegungen stellen die Entwicklung hin zu offenen Grenzen in Frage. Werte- und Leitkulturdebatten in allen europäischen Ländern und auch global, etwa in den USA und Indien, unterstützen das Streben nach Abgrenzung und ethnischer wie religiöser Homogenität einhergehend mit stärkerer Kontrolle der Grenzen. Diese Thematik wird erst ansatzweise von der Forschung aufgearbeitet (vgl. Casas-Cortes et al. 2013; Cassidy et al. 2018; Klatt 2018).

Ein weiterer Schwerpunkt zukünftiger Forschungen wird die funktionelle grenzüberschreitende Integration darstellen. Was bewirken grenzüberschreitende funktionelle Ströme und daraus entstehende Abhängigkeiten? Diese können durchaus zu einer funktionellen Spezialisierung führen, welche von sozialen und wirtschaftlichen Ungleichheiten abhängt, zum Beispiel einem deutlich niedrigeren Lohnniveau auf einer Seite der Grenze (vgl. Durand et al. 2017). Für die Analyse und Typisierung der institutionellen Zusammenarbeit, insbesondere auch innerhalb eines EVTZ, ist eine Weiterentwicklung des Konzepts der multi-level governance nötig (siehe dazu Ulrich/Scott in diesem Band). Wir wissen zu wenig über die nach wie vor vorhandenen Hierarchien des Mehrebenenregierungssystems, insbesondere in grenzüberschreitender Perspektive. Administrative, systemische und juristische Barrieren werden in allen grenzübergreifenden Regionen thematisiert. Für die Forschung über diese Thematiken wäre eine größere Interdisziplinarität wünschenswert. Ein weites Feld ist auch die Forschung über Grenzregionen als Lebensraum. Hier gibt es vereinzelte Pionierstudien, die sich mit verschiedenen Aspekten in unterschiedlichen Grenzregionen befassen. Es fehlt aber eine bessere Vernetzung dieser Studien, die Ausweitung auf periphere, dünn besiedelte Grenzregionen, insbesondere in Zentralund Osteuropa, und eine verstärkte Einbeziehung regionalökonomischer und auch betriebswirtschaftlicher Forschung zu diesen Fragestellungen.

\section{Weiterführende Literatur}

Beck, Joachim (Hrsg.) (2019): Transdisciplinary Discourses on Cross-Border Cooperation in Europe. Brüssel: Peter Lang.

Boesen, Elisabeth/Schnuer, Gregor (Hrsg.) (2017): European Borderlands. Living with Barriers and Bridges. Milton Park/New York: Routledge.

Durand, Frédéric/Decoville, Antoine/Knippschild, Robert (2017): Everything All Right at the EU Borders? The Ambivalent Effects of Cross-Border Integration and the Rise of Euroscepticism. In: Geopolitics. DOI: 10.1080/14650045.2017.1382475.

Krzymuski, Marcin/Kubicki, Philipp/Ulrich, Peter (2017): Der Europäische Verbund für territoriale Zusammenarbeit: Instrument der grenzübergreifenden Zusammenarbeit nationaler öffentlicher Einrichtungen in der Europäischen Union. Baden-Baden: Nomos.

Wastl-Walter, Doris (Hrsg.) (2011): The Ashgate Research Companion to Border Studies. Farnham/Burlington: Ashgate. 


\section{Literaturverzeichnis}

Aldecoa, Francesco/Keating, Michael (Hrsg.) (1999): Paradiplomacy in Action. The Foreign Relations of Subnational Governments. London/Portland: Frank Cass.

Andersen, Dorte Jagetic/Sandberg, Marie (2012): Introduction. In: Andersen, Dorte Jagetic/Klatt, Martin/ Sandberg, Marie (Hrsg.): The Border Multiple. The Practicing of Borders between Public Policy and Everyday Life in a Re-Scaling Europe. Farnham: Ashgate, S. 1-19.

Anderson, James/O'Dowd, Liam (1999): Borders, Border Regions and Territoriality: Contradictory Meanings, Changing Significance. In: Regional Studies 33, H. 7, S. 593-604.

Anderson, Malcolm (1996): Frontiers, Territory and State Formation in the Modern World. Oxford: Blackwell.

Banse, Christian (2004): Die Grenzregion. In: Ders./Stobbe, Holk (Hrsg.): Nationale Grenzen in Europa. Frankfurt/M.: Peter Lang, S. 35-52.

Baud, Michiel/Schendel, Willem van (1997): Towards a Comparative History of Borderlands. In: Journal of World History 8, H. 2, S. 211-242.

Blatter, Joachim (2004): From „Spaces of Place“ to „Spaces of Flow“? Territorial and Functional Governance in Cross-border Regions in Europe and North America. In: International Journal of Urban and Regional Research 28, H. 3, S. 530-548.

Böhm, Hynek/Drápela, Emil (2017): Cross-border cooperation as a reconciliation tool: Example from the East Czech-Polish borders. In: Regional \& Federal Studies 27, H. 3, S. 305-319.

Boesen, Elisabeth/Schnuer, Gregor (Hrsg.) (2017): European Borderlands. Living with Barriers and Bridges. Milton Park/New York: Routledge.

Brambilla, Chiara (2015): Exploring the Critical Potential of the Borderscapes Concept. In: Geopolitics 20, H. 1, S. 14-34.

Brambilla, Chiara/Laine, Jussi/Scott, James Wesley/Bocchi, Gianluca (Hrsg.) (2015): Borderscaping: Imaginations and Practices of Border Making. Farnham: Ashgate.

Brunet-Jailly, Emmanuel (2005): Theorizing Borders: An Interdisciplinary Perspective. In: Geopolitics 10, H. 4, S. 633-649.

Casas-Cortes, Maribel/Cobarrubials, Sebastian/Pickles, John (2013): Re-bordering the neighbourhood: Europe's emerging geographies of non-accession integration. In: European Urban and Regional Studies 20, H. 1, S. 37-58.

Cassidy, Katryn/Yuval-Davis, Nira/Wemyss, Georgie (2018): Debordering and everyday (re)bordering in and of Dover: Post-borderland borderscapes. In: Political Geography 66, S. 171-179.

Danish-German Transport Commission (2015): Transport Infrastructure in the Jutland Corridor. Copenhagen: The Danish Ministry of Transport and Building.

Donnan, Hastings/Wilson, Thomas M. (1999): Borders. Frontiers of Identity, Nation and State. Oxford/New York: Berg.

Durá, Antoni/Camonita, Francesco/Berzi, Matteo/Noferini, Andrea (2018): Euroregions, Excellence and Innovation across EU borders. A Catalogue of Good Practices. Barcelona: Department of Geography, UAB.

Durand, Frédéric/Decoville, Antoine/Knippschild, Robert (2017): Everything All Right at the EU Borders? The Ambivalent Effects of Cross-Border Integration and the Rise of Euroscepticism. In: Geopolitics 25, H. 3, S. 587-608.

ESPON (Hrsg.) (2018): Cross-border Public Services (CPS). Targeted Analysis. Final Report. Luxembourg: ESPON.

European Commission (2015): Cross-border Cooperation in the EU. Flash Eurobarometer. Brüssel.

Fitjar, Rune Dahl (2010): Explaining variation in sub-state regional identities in Western Europe. In: European Journal of Political Research 49, H. 4, S. 522-544.

Heddebaut, Odile (2004): The EUROREGION from 1991 to 2020: an ephemeral stamp? In: Kramsch, Olivier/Hooper, Barbara (Hrsg.): Cross-Border Governance in the European Union. London/New York: Routledge, S. 70-87.

Hobsbawm, Eric/Ranger, Terence (Hrsg.) (1983): The Invention of Tradition, Cambridge: Cambridge University Press.

Hroch, Miroslav (2005): Das Europa der Nationen. Die moderne Nationalitätenbildung im europäischen Vergleich. Göttingen: Vandenhoek \& Ruprecht.

Keating, Michael (Hrsg.) (2004): Regions and Regionalism in Europe. Cheltenham: Edward Elgar.

Klatt, Martin (2005): Die Minderheiten im deutsch-dänischen Grenzland in der grenzüberschreitenden Zusammenarbeit seit 1945. In: Kühl, Jørgen/Bohn, Robert (Hrsg.) Ein europäisches Modell? Nationale Minderheiten im deutsch-dänischen Grenzland 1945-2005. Bielefeld: Verlag für Regionalgeschichte, S. 306-323. 
Klatt, Martin (2006): Common, Cross-Border Regional History as an Approach to People-to-People Cooperation and Cross-Border Regional Integration. In: Hurd, Madeleine (Hrsg.): Borderland Identities. Territory and Belonging in Central, North and East Europe. Eslöv: Gondolin, S. 109-146.

Klatt, Martin (2013): National minorities as a model for cross-border integration. Lessons from Schleswig. In: Beck, Joachim/Wassenberg, Birte (Hrsg.): Grenzüberschreitende Zusammenarbeit leben und erforschen. Integration und (trans-)regionale Identität. Beiträge aus dem Kolloquium „Grenzen überbrücken: auf dem Weg zur territorialen Kohäsion in Europa“, 18./19. Oktober 2010, Straßburg. Stuttgart: Franz Steiner, S. 301-320.

Klatt, Martin (2014): (Un)Familiarity? Labor Related Cross-Border Mobility in Sønderjylland/Schleswig Since Denmark Joined the EC in 1973. In: Journal of Borderlands Studies 29, S. 353-373.

Klatt, Martin (2017): Minorities as secondary foreign policy agents in peace-building and reconciliation? The case of Denmark and Germany. In: Regional \& Federal Studies 27, S. 239-259.

Klatt, Martin (2018): The So-Called 2015 Migration Crisis and Euroscepticism in Border Regions: Facing Re-Bordering Trends in the Danish-German Borderlands. In: Geopolitics. DOI: 10.1080/14650045.2018.1557149.

Klatt, Martin/Herrmann, Hajo (2011): Half Empty or Half Full? Over 30 Years of Regional Cross-Border Cooperation Within the EU: Experiences at the Dutch-German and Danish-German Border. In: Journal of Borderlands Studies 26, S. 65-87.

Klatt, Martin/Kühl, Jørgen: (2008): National Minorities and Crossborder Cooperation between Hungary and Croatia. A Case Study of Baranya, Hungary, and Osijecko-baranjska County, Croatia. In: European Yearbook of Minority Issues 6, S. 193-210.

Klatt, Martin/Wassenberg, Birte (2018): Secondary foreign policy: Can local and regional cross-border cooperation function as a tool for peace-building and reconciliation? In: Dies. (Hrsg.): Secondary Foreign Policy in Local International Relations. Peace-Building and Reconciliation in Border Regions. London/New York: Routledge, S. 1-14.

Kolossov, Vladimir/Scott, James W. (2013): Selected conceptional issues in border studies. In: Belgeo (online). Revue belge géographique. DOI 10.4000/belgeo.10532.

Lepik, Krista L. (2009): Euroregions as Mechanisms for Strengthening Cross-Border Cooperation in the Baltic Sea Region. In: Trames-Journal of the Humanities and Social Sciences 13, S. 265-284.

Lewandowski, Krzysztof/Greta, Maria: (2010): Euroregion’s „mission“ and the success of the Lisbon strategy. In: Business and Economic Horizons 1, S. 14-20.

Löfgren, Orvar (2008): Regionauts: the Transformation of Cross-Border Regions in Scandinavia. In: European Urban and Regional Studies 15, S. 195-209.

Makkonen, Teemu/Williams, Alan M./Weidenfeld, Adi/Kaisto, Virpi (2018): Cross-border knowledge transfer and innovation in the European neighbourhood: Tourism cooperation at the Finnish-Russian border. In: Tourism Management 68, S. 140-151.

Malloy, Tove (2010): Creating New Spaces for Politics? The Role of National Minorities in Building Capacity of Cross-Border Regions. In: Regional \& Federal Studies 20, S. 335-351.

Martinez, Oscar J. (1994): Border People. Life and Society in the U.S.-Mexico Borderlands. Tucson/London: University of Arizona Press.

Medeiros, Eduardo (2011): (Re)defining the Euroregion Concept. In: European Planning Studies 19, S. 141-158.

Medve-Balint, Gergö/Svensson, Sara (2012): Explaining Coverage: Why do Local Governments in Central Europe join (or not join) Euroregions? In: Jagetic Andersen, Dorte/Klatt, Martin/Sandberg, Marie (Hrsg.): The Border Multiple. The Practicing of Borders Between Public Policy and Everyday Life in a Re-scaling Europe. Farnham: Ashgate, S. 219-243.

Naveed, Amjad/Ahmad, Nisar (2016): Technology Spillovers and International Borders: A Spatial Econometric Analysis. In: Journal of Borderlands Studies, 31, S. 441-461.

Nerb, Gernot/Hitzelsberger, Franz/Woidich, Andreas/Pommer, Stefan/Hemmer, Sebastian/Heczko, Petr (2009): Scientific Report on the Mobility of Cross-border Workers in the EU-27/EEA/EFTA Countries. München/Sopron: MKW Wirtschaftsforschung GmbH/Empirica Kft.

O'Dell, Thomas (2003): Øresund and the Regionauts. In: European Studies: A Journal of European Culture, History and Politics 19, S. 31-53.

Ohmae, Kenichi (1995): The End of the Nation State. The Rise of Regional Economies. London: Harper Collins.

Paasi, Anssi (2002): Bounded spaces in the mobile world: Deconstructing „regional identity“. In: Journal of Economic \& Social Geography 93, S. 137-148.

Paasi, Anssi (2009a): Bounded spaces in a „borderless world“: border studies, power and the anatomy of territory. In: Journal of Power 2, S. 213-234. 
Paasi, Anssi (2009b): The resurgence of the „region“ and „regional identity“: theoretical perspectives and empirical observations on regional dynamics in Europe. In: Review of International Studies 35 , S. 121-146.

Palermo, Francesco (2005): Trans-Border Cooperation and Ethnic Diversity. In: Kühl, Jørgen/Weller, Marc (Hrsg.) Minority Policy in Action: The Bonn-Copenhagen Declarations in a European Context 19552005. Aabenraa: Institut for Grænseregionsforskning, S. 161-186.

Parker, Noel/Vaughan-Williams, Nick (2009): Lines in the Sand? Towards an Agenda for Critical Border Studies. In: Geopolitics 14, S. 582-587.

Perkmann, Markus (2002): Euroregions: Institutional Entrepreneurship in the European Union. In: Perkmann, Markus/Sum, Ngai-Ling (Hrsg.): Globalization, Regionalization and Cross-Border Regions. Basingstoke/New York: Palgrave Macmillan, S. 103-124.

Perkmann, Markus (2003): Cross-Border Regions in Europe. Significance and Drivers of Regional CrossBorder Cooperation. In: European Urban \& Regional Studies, 10, S. 153-171.

Popescu, Gabriel (2008): The conflicting logics of cross-border reterritorialization: geopolitics of Euroregions in Eastern Europe. In: Political Geography 27, S. 418-438.

Ratzel, Friedrich (1901/2017): The Laws of the Spatial Growth of States. In: Kasperson, Roger E./Minghi, Julian (Hrsg.): The Structure of Political Geography. New York: Routledge, S. 17-28.

Rumford, Charles (2008): Introduction: Citizens and Borderwork in Europe. In: Space and Polity 12, H. 1, S. 1-12.

Schmitt-Egner, Peter (1998): „Grenzüberschreitende Zusammenarbeit“ in Europa als Gegenstand wissenschaftlicher Forschung und Strategie transnationaler Praxis. Anmerkungen zur Theorie, Empirie und Praxis des Transnationalen Regionalismus. In: Brunn, Gerhard/Schmitt-Egner, Peter (Hrsg.): Grenzüberschreitende Zusammenarbeit in Europa. Theorie - Empirie - Praxis. Baden Baden: Nomos, S. 27-77.

Scott, James W. (2000): Euroregions, Governance, and Transborder Cooperation Within the EU. In: Velde, Martin van der/Houtum, Henk van (Hrsg.): Borders, Regions and People. London: Pion, S. 104-115.

Scott, James W. (2013): Constructing Familiarity in Finnish-Russian Karelia: Shifting Uses of History and the Re-Interpretation of Regions. In: European Planning Studies 21, H. 1, S. 75-92.

Sohn, Christophe (2014): The Border as a Resource in the Global Urban Space: A Contribution to the Cross-Border Metropolis Hypothesis. In: International Journal of Urban and Regional Research 38, H. 5, S. 1697-1711.

Sohn, Christophe/Lara-Valencia, Francisco (2013): Borders and Cities: Perspectives from North America and Europe. In: Journal of Borderlands Studies, 28, H. 2, S. 181-190.

Spierings, Bas/Velde, Martin van der (2008): Shopping, Borders and Unfamiliarity: Consumer Mobility in Europe. In: Journal for Economic and Social Geography 99, H. 4, S. 497-505.

Spierings, Bas/Velde, Martin van der (2013): Cross-Border Differences and Unfamiliarity: Shopping Mobility in the Dutch-German Rhine-Waal Euroregion. In: European Planning Studies 21, H. 1, S. 5-23.

Terlouw, Kees (2008): The Discrepancy in PAMINA between European image of a cross-border region and cross-border behaviour. In: GeoJournal 73, S. 103-116.

Terlouw, Kees (2012): Border Surfers and Euroregions: Unplanned Cross-Border Behaviour and Planned Territorial Structures of Cross-Border Governance. In: Planning Practice and Research 27, H. 3, S. 351366.

Topaloglou, Lefteris/Kallioras, Dimitis/Manetos, Panos/Petrakos, George (2005): A Border Regions Typology in the Enlarged European Union. In: Journal of Borderlands Studies, 20, H. 2, S. 67-90.

Varró, Krisztina/Lagendijk, Arnoud (2012): Conceptualizing the Region - In What Sense Relational? In: Regional Studies 47, H. 1, S. 18-28.

Wille, Christian (Hrsg.) (2015): Lebenswirklichkeiten und politische Konstruktionen in Grenzregionen: das Beispiel der Großregion SaarLorLux: Wirtschaft - Politik - Alltag - Kultur. Bielefeld: transcript.

Yndigegn, Carsten (2012): Reviving Unfamiliarity-The Case of Public Resistance to the Establishment of the Danish-German Euroregion. In: European Planning Studies, 21, H. 1,S. 58-74.

Zahra, Tara (2010): Imagined Noncommunities: National Indifference as a Category of Analysis. In: Slavic Review 69, H. 1, S. 93-119.

Zumbusch, Kristina/Scherer, Roland (2015): Cross-Border Governance: Balancing Formalized and Less Formalized Co-Operations. In: Social Sciences 4, H. 3, S. 499-519. 